\title{
A WUSCHEL-related homeobox 3B gene, depilous (dep), confers glabrousness of rice leaves and glumes
}

\author{
Rosalyn B Angeles-Shim ${ }^{1,3}$, Kenji Asano 1,4, Tomonori Takashi ${ }^{2}$, Junghyun Shim ${ }^{1,3}$, Takeshi Kuroha ${ }^{1}$, \\ Madoka Ayano ${ }^{1}$ and Motoyuki Ashikari ${ }^{1 *}$
}

\begin{abstract}
Background: Glabrousness is an important agricultural trait for the practical breeding of rice. In this study, depilous (dep), the gene responsible for glabrous leaves and glumes of rice was identified by map-based cloning.

Results: The dep gene encodes a WUSCHEL-related homeobox 3B that was fine-mapped to a 22-kb region on the short arm of chromosome 5 using progenies derived from crosses between Koshihikari (pubescent) and GLSL15, an Oryza glaberrima chromosome segment substitution line (glabrous). Complementation tests confirmed the conditioning of the glabrous phenotype by the dep gene. Phylogenetic analysis showed that dep groups with the WOX3 family of plant-specific homeobox transcription factors that are involved in regulating lateral organ development. Localization of dep in the nucleus indicates the function of the gene as a transcription factor. Spatial expression of the gene was observed in the base of young shoots, the leaf sheath, midrib, young roots and nodal structures.

Conclusion: The identification and cloning of dep will not only provide basis for future research on the elucidation of the molecular mechanisms underlying trichome formation in rice but will also aid in breeding programs for the development of glabrous varieties.
\end{abstract}

Keywords: depilous, Rice, Trichomes, Glabrous, Leaves, Glumes, WUSCHEL-related homeobox

\section{Background}

Trichomes are specialized structures that originate from the aerial epidermis and develop as hair-like projections that extend from the epidermal surface (Serna and Martin 2006; Werker 2000; Johnson 1975). They differ considerably in cellular composition, density, morphology, location and, depending on whether they are glandular or nonglandular, in function. In most plant species, nonglandular trichomes function to lessen the heat load of leaves, reduce transpiration rates, and enhance freezing tolerance and herbivore resistance. Glandular trichomes on the other hand, not only offer physical resistance but also provide chemical protection against biotic and abiotic challenges (Serna and Martin 2006; Martin and Glover

\footnotetext{
* Correspondence: ashi@agr.nagoya-u.ac.jp

${ }^{1}$ Bioscience and Biotechnology Center, Nagoya University, Chikusa, Nagoya Aichi 464-8601, Japan

Full list of author information is available at the end of the article
}

2007; Bandyopadhyay et al. 2004; Werker 2000; Mauricio and Rauscher 1997; Johnson 1975).

In rice (Oryza sativa), trichomes on the leaf blade take the form of hairs or bristles (Yamamoto et al. 1997; Hoshikawa 1975). The hairs are two-celled, cylindrical appendages that are arranged between nerves on the epidermis on either side of the leaves. Bristle type trichomes on the other hand, are one- or two-celled outgrowths, and may be of type Ia, Ib or II. The bristle type Ia trichomes are commonly found on the adaxial side of the leaf and are slightly longer than the type Ib. Both the hair and the bristle type II trichomes are located on the adaxial and abaxial leaf surfaces. The bristle type Ia and Ib trichomes as well as the hairs run perpendicular to the leaf surface, whereas the bristle type II are oriented parallel to the leaf blade. Both bristle type Ia and Ib trichomes are conical in shape despite differences in size and location, indicating that they may have

\section{实}

(c) 2012 Angeles-Shim et al.; licensee Springer. This is an Open Access article distributed under the terms of the Creative Commons Attribution License (http://creativecommons.org/licenses/by/2.0), which permits unrestricted use, distribution, and reproduction in any medium, provided the original work is properly cited. 
been derived from the same origin (Yamamoto et al. 1997). The bristle type trichomes, regardless of length or orientation, have also been described as prickle hairs (Islam et al. 2009) or macrohair, whereas the hair has also been referred to as microhair (Kobayashi et al. 1997; Shimizu et al. 1976).

The reduction or absence of the bristle type trichomes on the surface of the leaves and the glumes defines the glabrous phenotype in rice. This trait has been considered as selectively neutral, with neither a known selective advantage nor disadvantage. However, in areas where rice culture remains manual, the glabrous trait is fairly valued since the lack of trichomes reduces skin irritation and itching among workers during harvesting, threshing, drying and milling of rice (Rutger and Mackill 2001; Makenzie et al. 1987). In the U.S., cultivation of glabrous varieties has been an important part of the rice industry. Initially valued for hand harvesting and threshing because they cause less itching, the glabrous varieties became more prized due to their higher bulk density compared with pubescent varieties (Rutger and Mackill 2001). The higher bulk density due to the close packing of the glabrous kernels saves valuable space during storage and transportation (Varnamkhasti et al. 2008; Rutger and Mackill 2001). Glabrous varieties have also been known to create less dust during processing. At present, virtually all U.S. rice varieties, as well as many Australian varieties that share U.S. parentage, are glabrous (Rutger and Mackill 2001).

The recessive allele $g l 1$ and its duplicate pair $g l 2$ have been reported to control glabrousness in both the leaves and glumes of rice (Yamamoto et al. 1997; Nagao and Takahashi 1963; Sastry and Seetharaman 1980; Sato et al. 1990). Linkage maps associated $g l 1$ with restriction fragment length polymorphism markers mapped on

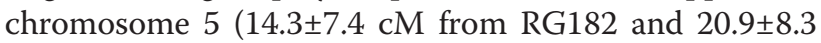
cM from RG403) ( $\mathrm{Yu}$ et al. 1992; $\mathrm{Yu}$ et al. 1995). To date, however, the gene controlling the trait has yet to be identified and characterized. We report here the identification and cloning of depilous (dep), the gene regulating trichome formation in rice leaves and glumes. This gene was identified by positional cloning using a previously identified glabrous chromosome segment substitution line (GLSL15) carrying an introgressed fragment from the African cultivated rice, O. glaberrima in the background of O. sativa subsp. japonica cv. Koshihikari. dep encodes a WUSCHEL-related homeobox 3B protein that belongs to a class of transcription factors regulating lateral organ development.

\section{Results}

Phenotype of the substitution line, GLSL15

The substitution line GLSL15 shares the genomic constitution of cv. Koshihikari except for an approximately
3.15-Mb fragment of $O$. glaberrima on the short arm of chromosome 5 (Figure 1a-c). Gross morphological examination showed that GLSL15 closely resembles Koshihikari (Figure 1d-f) although scanning electron micrographs revealed that the substitution line is more similar to $O$. glaberrima in leaf ultrastructure (Figure 1g-1). Both GLSL15 and O. glaberrima lack the bristle-type trichomes and have hairs only on both the abaxial and adaxial surface of the leaves. The hairs run parallel to the leaf venation and are located beside the motor cells or stomata. Koshihikari leaves, on the other hand, have both hair and the bristle-type trichomes. Bristle type Ia, Ib and II trichomes were observed on the adaxial leaf surface, whereas only bristle type Ia and Ib were seen on the abaxial leaf surface. The bristle-type trichomes are located on silica cells over a thin vascular bundle and are oriented perpendicular or parallel to the leaf blade. Trichomes that are prominent on the glumes of Koshihikari are absent on the glumes of both GLSL15 and O. glaberrima (Figure 1m-o).

\section{Genetic analysis and fine-scale mapping of the dep gene} Linkage analysis using $600 \mathrm{~F}_{2}$ lines generated from crosses between Koshihikari and GLSL15 showed that the gene controlling glabrousness in rice, herein named depilous (dep), is tightly linked to SSR markers RM159 (0-2.3 cM) and RM13 (28.6-31.4 cM) on the short arm of chromosome 5 (Figure 2a). Fine mapping of the dep gene using the same $600 \mathrm{~F}_{2}$ lines and newly developed indel-based and dCAPs markers narrowed down the candidate region to $175 \mathrm{~kb}$. This region was further delimited to $62 \mathrm{~kb}$ by fine mapping using an initial set of $1152 \mathrm{~F}_{3}$ plants generated from the $\mathrm{F}_{2}$ lines with informative recombination points. Genotyping of an additional $6500 \mathrm{~F}_{3}$ lines finally identified a 22-kb candidate region for the gene (Figure 2b). This 22-kb stretch is covered in a single BAC (P0496H07) and includes 4 annotated genes encoding the ORF for a retrotransposon protein (LOC_Os5g02710), a hypothetical protein (LOC_Os05g02720), a WUSCHELrelated homeobox 3B (LOC_Os05g2730) and an expressed protein (LOC_Os05g2740) (Figure 2c). The observed Mendelian ratio for pubescent and glabrous types in the $F_{2}$ population indicates a monogenic control and recessive inheritance of the trait although a slight segregation distortion was observed (data not shown).

\section{Map-based cloning of the dep gene}

To determine which of the candidate genes regulate trichome formation in rice, a series of complementation tests were carried out parallel to the fine-scale mapping. Six overlapping fragments covering the initially identified 62 $\mathrm{kb}$ candidate region (Figure $3 \mathrm{a}$ ) were picked from a newly developed sub-library of a Koshihikari BAC clone. From these, 2 overlapping fragments spanning 30-kb (LL3) and 

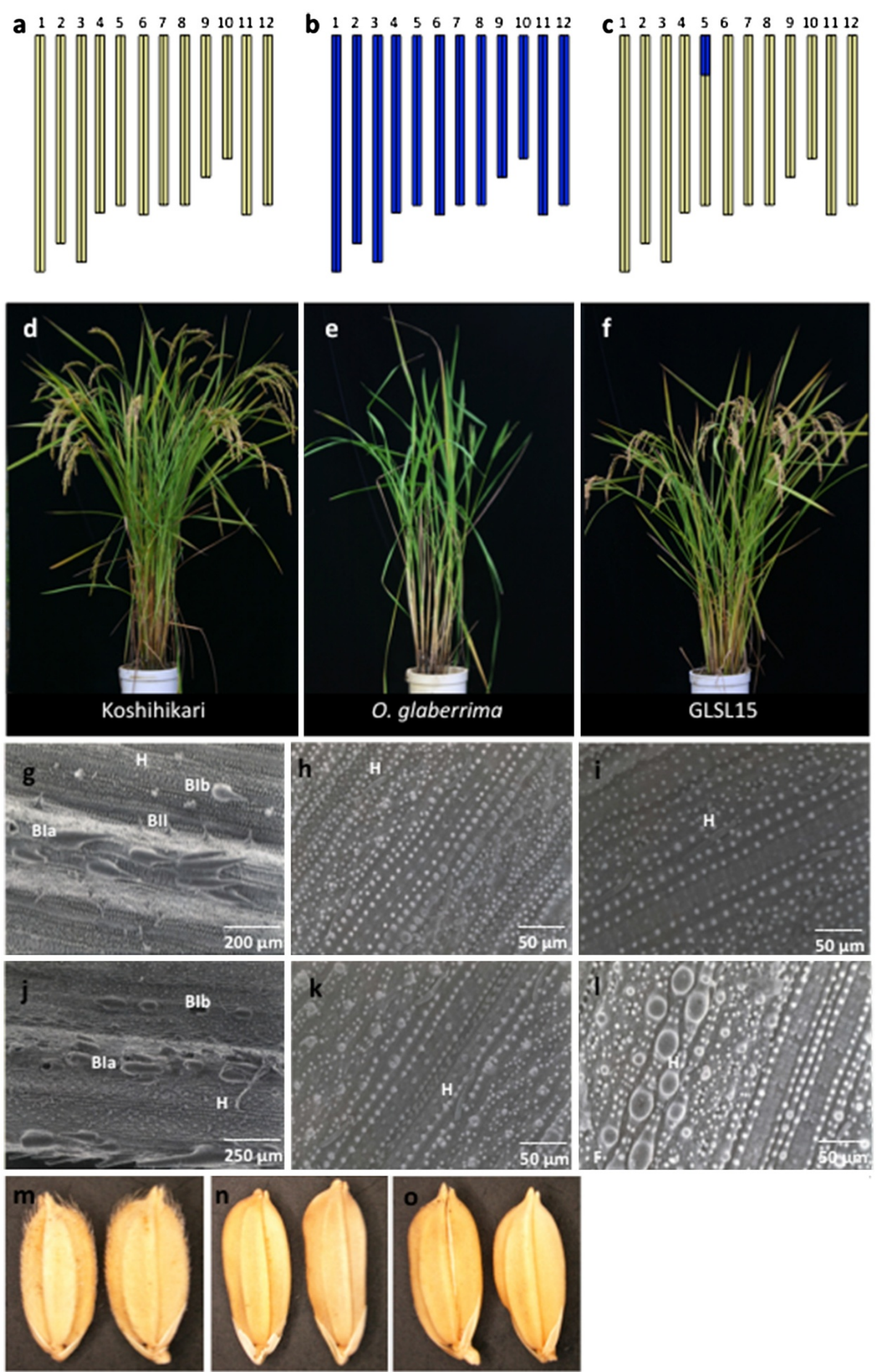

Figure 1 (See legend on next page.) 
(See figure on previous page.)

Figure 1 Morphology and graphical genotypes of the plant materials. Graphical representations of the genomic constitution of (a) Koshihikari, (b) O. glaberrima and (c) GLSL15. Gross morphology and overall structural variation among (d) Koshihikari (e) O. glaberrima (f) and GLSL15 during the reproductive stage. Scanning electron micrographs of the adaxial and abaxial leaf surfaces of ( $\mathbf{g}, \mathbf{j})$ Koshihikari, (h, k) $O$. glaberrima and (i, I) GLSL15. (m) Koshihikari showing trichomes in the glumes, and (n) O. glaberrima and (o) GLSL15 exhibiting glabrousness or absence of trichomes on the glumes. $\mathrm{H}=$ hair, Bla=bristle type la, Blb-bristle type $\mathrm{lb}, \mathrm{B} \|=$ bristle type $\mathrm{I}$.

$15-\mathrm{kb}$ (LL5) restored the pubescent phenotype in a glabrous substitution line that was used for transformation (Figure $3 \mathrm{~b}$ ). $\mathrm{T}_{0}$ plants carrying the LL3 or LL5 fragment showed bristle-type trichomes on both adaxial and abaxial leaf surfaces and also on the glumes of the spikelets. $\mathrm{T}_{1}$ plants of both LL3 and LL5 also had the same pubescent phenotype. Using the LL3 sub-clone, 5 overlapping fragments were generated and used for another round of complementation tests. From the 5 fragments, sub-clones LL3scUL6 and LL3scG2, spanning 20 and $11 \mathrm{~kb}$, respectively, recovered the pubescent phenotype of the glabrous substitution line used (Figure $3 \mathrm{c}$ ). $\mathrm{T}_{0}$ plants carrying the LL3scUL6 or LL3scG2 fragments had the bristle type trichomes on both the adaxial and abaxial leaf surface, as well as on the glumes. The LL3scG2 genomic fragment included the annotated open reading frames (ORFs) for LOC_Os05g02710, LOC_Os05g2720 and LOC_Os05g2730 (Figure $3 \mathrm{~d}$ ). A third and final complementation test used the LL3scG2 sub-clone to generate 5 overlapping fragments i.e. LL3scG2a, LL3scG2b, LL3scG2e, LL3scG2g and LL3scG2c for transformation (Figure 3e). From these, only LL3scG2a, LL3scG2c and L3scG2g spanning 3.5, 6 and $3.2 \mathrm{~kb}$, respectively, restored the pubescent phenotype of the substitution line used for the complementation analysis. Transgenic plants carrying any of these genomic fragments showed bristle type trichomes on both adaxial

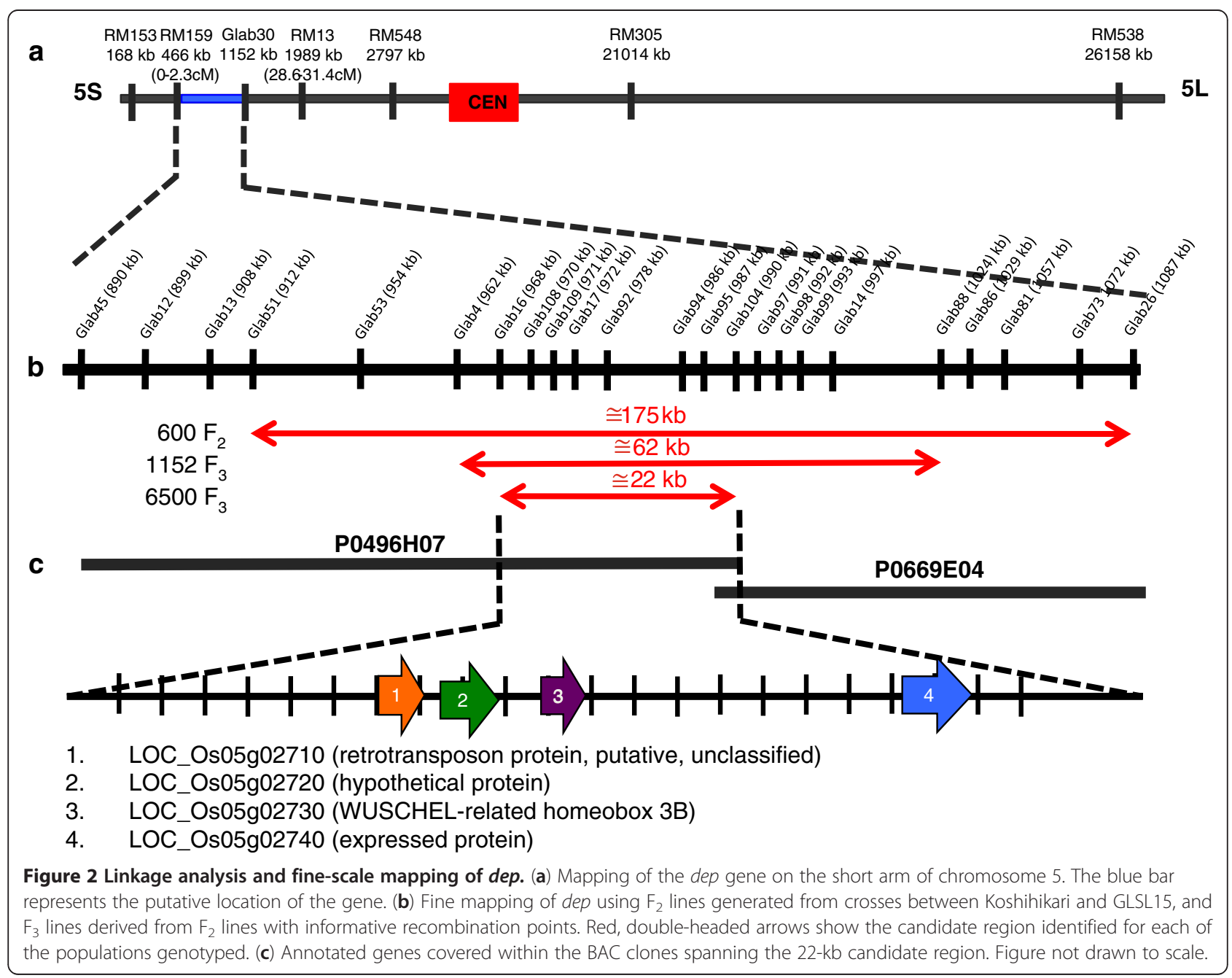




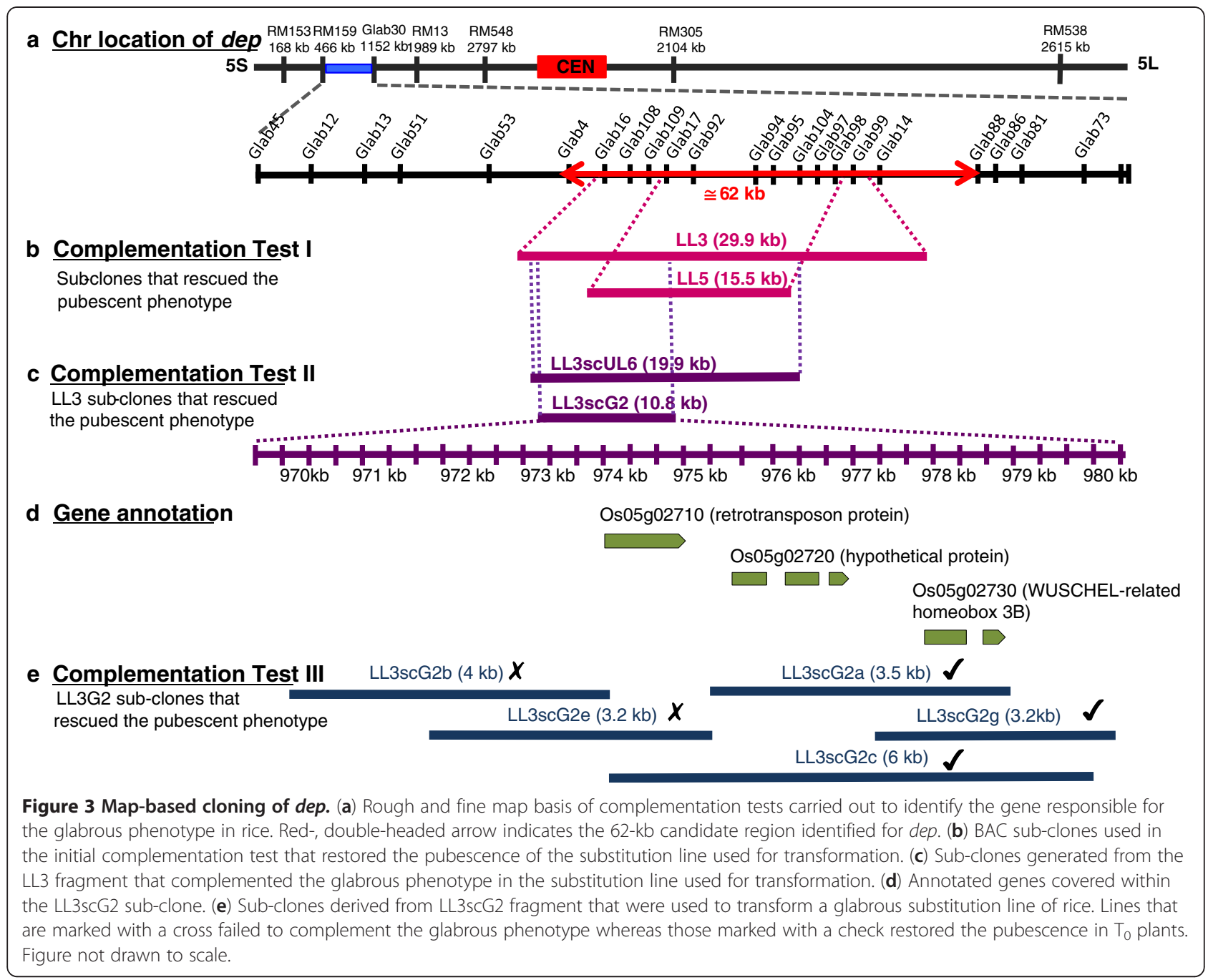

and abaxial leaf surfaces, as well as on the glumes (Figure 4). LL3scG2c covered the ORFs for the three candidate genes, whereas LL3scG2a included only the ORFs for LOC_Os05g2720 and LOC_Os05g2730. The LL3scG2g sub-clone covered only the ORF of LOC_Os05g2730 encoding a WUSCHEL-related homeobox 3B. These results indicate that LOC_Os05g2730 is the dep gene that conditions glabrousness in rice.

\section{Structure, phylogeny and comparative sequence analysis of dep encoding a WUSCHEL-related homeobox 3B protein}

Combined fine mapping and complementation tests demonstrated that LOC_Os05g2730 corresponding to the dep gene regulates the glabrous phenotype in rice. The gene is $861 \mathrm{bp}$ in length and encodes a WUSCHELrelated homeobox 3B protein composed of 286 amino acids (Figure 5a). The dep protein is characterized by a homeobox domain in the $\mathrm{N}$ terminal and a WUS box motif in the $\mathrm{C}$ terminal. Amino acid sequences of both the homeobox domain and WUS box motif are highly conserved in dep and in the WUSCHEL HOMEOBOX (WOX) 3 orthologues in Arabidopsis thaliana, maize (Zea mays) and sorghum (Sorghum bicolor) (Figure 5b). Phylogenetic analysis using $A$. thaliana WOX 10 as outgroup showed that dep groups with other WOX3 relatives from $A$. thaliana, maize and sorghum, although it roots outside the Arabidopsis WOX3/PRESSED FLOWER (PRS)-maize Narrow Sheath (NS) branch (Figure 5c).

Sequencing of dep showed 3 amino acid substitutions (G23V, V14A and T34A) and a serine deletion (S32del) in the O. glaberrima allele relative to the Koshihikari allele. To determine which of these mutations causes the glabrous phenotype, sequencing of dep in glabrous rice mutants, as well as in a natural population of pubescent and glabrous $O$. glaberrima lines was also carried out. Sequence analysis showed that both glabrous and pubescent lines of $O$. glaberrima carry the same amino acid substitutions and deletion relative to Koshihikari. In 


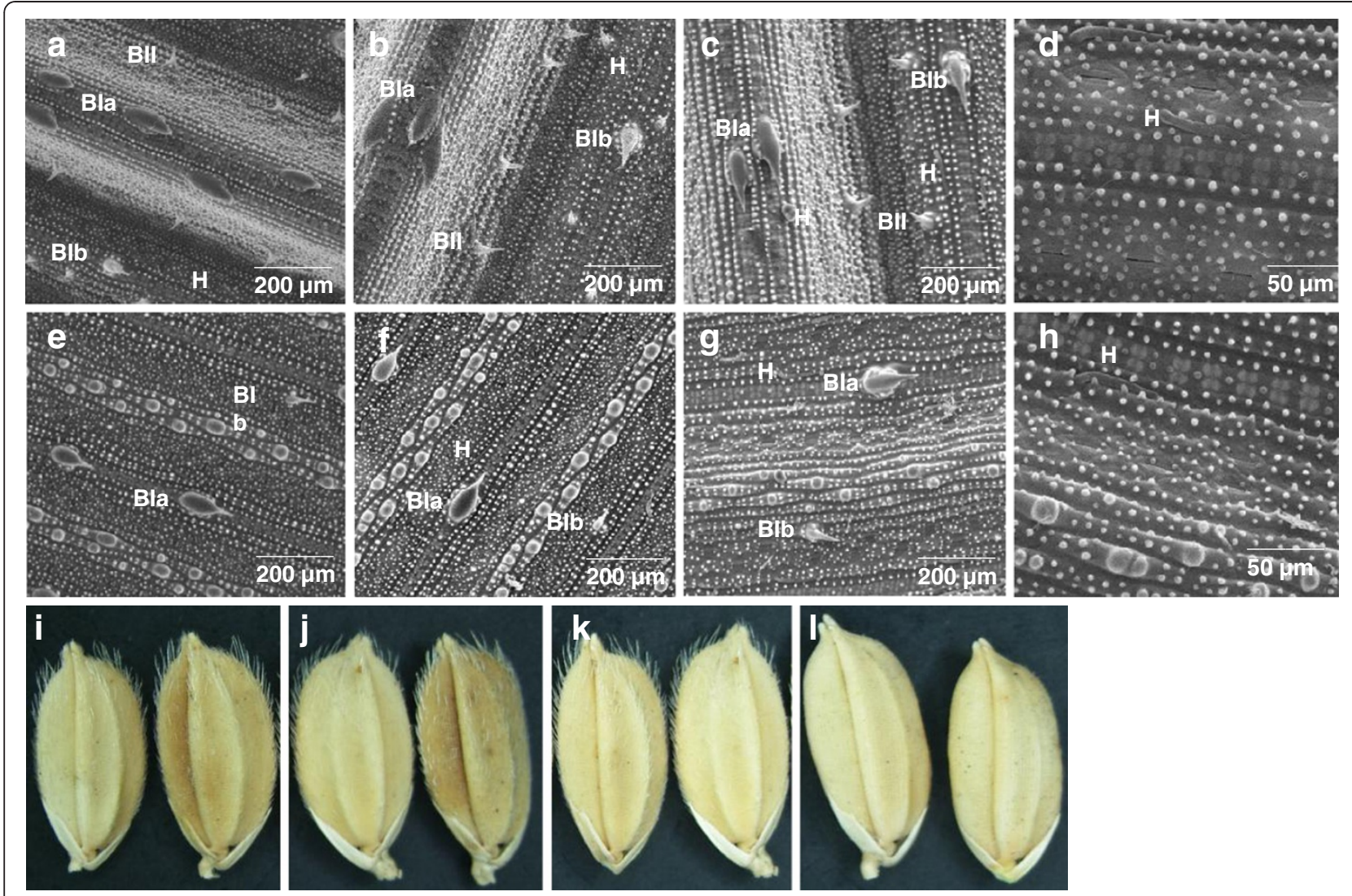

Figure 4 Restoration of the pubescent phenotype in a glabrous substitution line (cv. T65 background). Scanning electron micrographs of the adaxial and abaxial leaf surfaces of transgenic plants ( $\left.T_{0}\right)$ carrying the LL3scG2a (a, e), LL3scG2c (b, f) and LL3scG2g (c, g) genomic fragments, exhibiting recovery of bristle type trichomes. Hairy glumes of transgenic plants ( $\left.T_{0}\right)$ carrying the (i) LL3scG2a, (j) LL3scG2c and (k) LL3scG2g genomic fragments. Scanning electron micrographs of the leaves of the vector control showing absence of trichomes on both the (d) adaxial and (h) abaxial sides of the leaf, as well as on the (I) glumes. $\mathrm{H}=$ hair, Bla=bristle type la, Blb-bristle type Ib, Bll=bristle type II.

addition, dep sequence in the glabrous rice mutants studied was similar to that of Koshihikari. Together, these results indicate that none of the sequence variations observed is responsible for the glabrous trait in rice. Sequencing of the $2-\mathrm{kb}$ region upstream of $\mathrm{dep}$ also did not show any difference between O. glaberrima and Koshihikari.

\section{Subcellular localization and spatial expression of dep}

Transient expression in onion epidermal cells of a translational fusion protein between dep and a GFP reporter gene showed that the gene localizes in the nucleus (Figure 6). GUS activity in transgenic plants expressing the $\beta$-glucuronidase reporter gene fused to a dep promoter fragment was detected in the shoot base, lateral roots and leaf sheath of 10-day-old plants and in the nodes and mid-vein of 20-day-old plants (Figure 7a-e).

\section{Discussion}

Glabrous mutants exhibiting a few or total lack of trichomes in the leaves have been reported for a wide range of species. In $A$. thaliana, considerable progress has been made in elucidating the molecular mechanisms involved in trichome initiation, morphogenesis and patterning (Szymanski et al. 2000). To date, the specification of trichome formation in Arabidopsis has been demonstrated to require the interaction of bHLH, MYB and WD repeat proteins in a functional complex (Zhao et al. 2008; Tominaga et al. 2008; Hauser et al. 2001). In cotton (Gossypium arboretum), similar protein complexes have been reported to control trichome formation. GaMYB2, an Arabidopsis GLABRA1 (GL1) orthologue in cotton, has been reported to have an amino acid motif for interaction with a bHLH protein. GaMYB2 expressed in A. thaliana under the control of GL1 promoter fully restored trichome formation in a gl1 mutant. GhMYB109 is another R2R3 MYB gene identified in upland cotton (G. hirsutum). Like GaMYB2, it also has a conserved motif for interaction with R-like bHLH proteins. Two other functional homologues of the Arabidopsis TRANSPARENT TESTA GLABRA were identified in cotton and both genes 


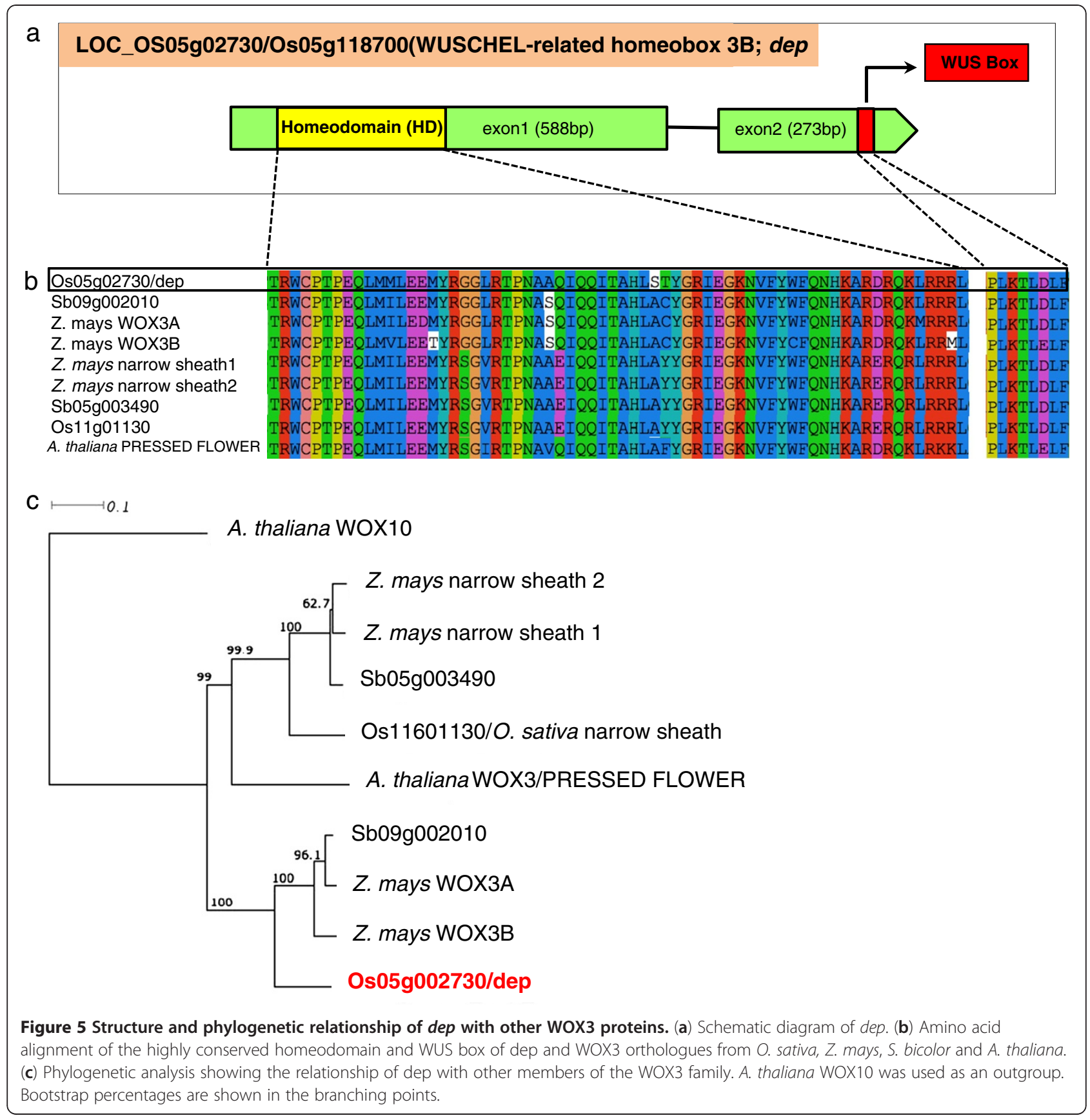

encode WD repeat proteins (Humphries et al. 2005; Wang et al. 2004; Suo et al. 2003; Serna and Martin 2006). Despite parallelisms in the model system for trichome regulation in Arabidopsis and cotton, similar multimeric complexes among MYB, bHLH and WD protein repeats have not been associated with trichome formation in species outside the Rosid division to which Arabidopsis and cotton both belong. In fact, different regulatory genes have been demonstrated to induce the formation of the various types of trichomes, as in the case of Asterids (Serna and Martin 2006). Furthermore, many plant species produce different types of trichomes that appear to be independently regulated (Moose et al. 2004).

In our study, dep, the gene responsible for the glabrous phenotype in rice, was mapped and identified by positional cloning. The rice substitution line, GLSL15 exhibits a glabrous phenotype that is characterized by the absence of trichomes on the leaf blade and the glumes. This line has the full genomic constitution of $\mathrm{cv}$. Koshihikari except for a 3.15-Mb stretch of chromosome 5 of O. glaberrima. Glabrousness in this substitution line 


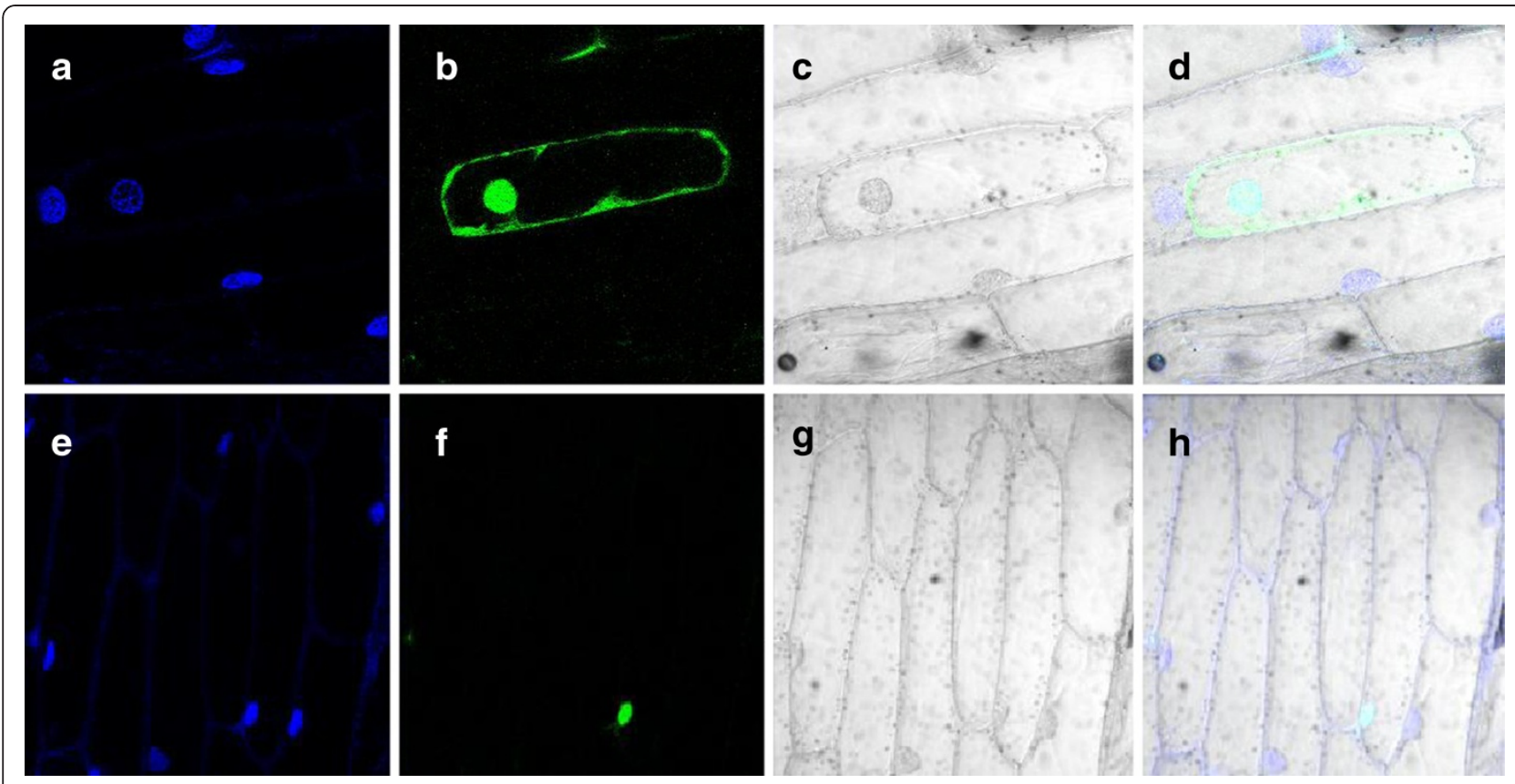

Figure 6 Subcellular localization of the dep-GFP protein in onion epidermal cells analyzed by confocal laser microscopy. DAPI stained, GFP, bright field and merged images of onion epidermal cells expressing 35S::GFP as a control (a-d) and 355::.dep-GFP (e-h).

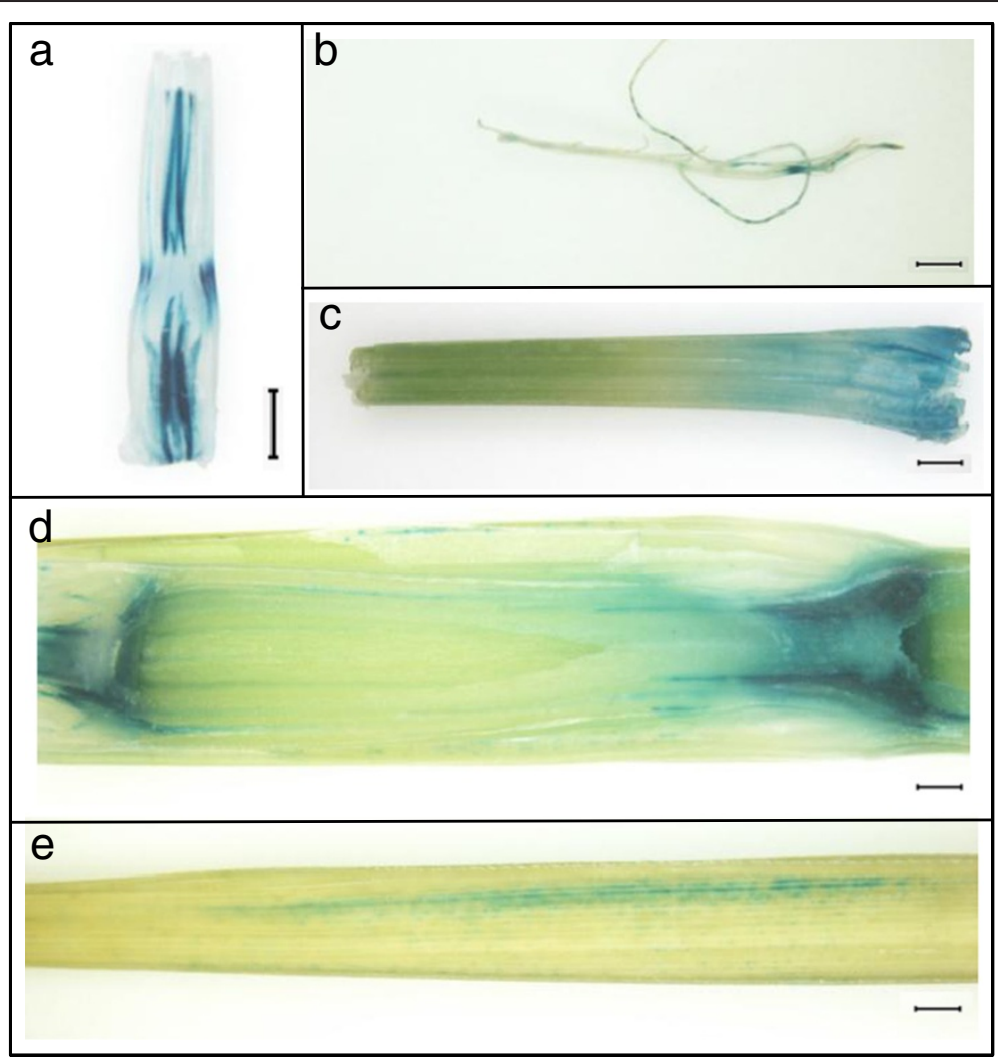

Figure 7 Histochemical GUS assay using various organs of rice transformants carrying dep promoter-GUS. GUS staining was observed in the (a) shoot base (longitudinal section), (b) lateral roots and (c) leaf sheath of 10-day-old plants as well as in the (d) nodal structures and (e) mid-vein of 20-day-old plants. Scale bar=1 mm. 
has been associated with the introgressed fragment of O. glaberrima (Shim et al. 2010).

Rough mapping of dep identified the location of the gene between 0-2.3 cM (RM159) and 28.6-31.4 cM (RM13) on the short arm of chromosome 5, coinciding with the reported rice $g l 1$ identified between 3.8 and $24.8 \mathrm{cM}$, also on the short arm of chromosome 5. Genotyping and phenotyping of a total of $8252 \mathrm{~F}_{2}$ and $\mathrm{F}_{3}$ lines showed a monogenic and recessive control of the trait similar to previous reports on the monogenic control of glabrousness (Li et al. 2010; Foster and Rutger 1978). A slight distortion in genetic segregation for the trait was observed and this may be due to the preferential fertilization of a particular gametic genotype. Segregation distortion has been frequently observed at the $g l 1$ locus in indica $\mathrm{x}$ glabrous japonica crosses and changes in the segregation ratio for $g l 1$ have been reportedly caused by the unequal chances of fertilization of the female gametes (Sato et al. 1990).

Fine-scale mapping narrowed the candidate region for dep into a 22-kb stretch covering 4 annotated genes that encode ORFs for a retrotransposon protein (LOC_05g02710), a hypothetical protein (LOC_05g02720), a WUSCHEL-related homoebox 3B (LOC_05g02730, dep) and an expressed protein (LOC_05g02740). In silico analysis showed that LOC_05g02710 contains a transposase DDE domain that has not been previously associated with any regulatory pathways for trichome formation. Blastp searches showed that LOC_05g02720 has a MYB/SANTlike DNA binding domain that has been reported to play regulatory roles in defense response and developmental processes in plants (Barg et al. 2005; Yanhui et al. 2006). In Arabidopsis, small MYB proteins including TRIPTYCHON (TRY), CAPRICE (CPC), TRICHOMELESS1 and ENHANCER OF TRY AND CPC 1, 2 and 3 have been identified as negative regulators of trichome initiation and patterning in the shoots (Wada et al. 1997; Kirik et al. 2004; Wang et al. 2008). However, such regulatory proteins have been associated with trichome formation in species only within the Rosid division (Serna and Martin 2006; Moose et al. 2004). LOC_05g02740 is a single-copy gene with a predicted protein length of 56 amino acids. Blastp searches indicate that orthologues of this gene in maize and sorghum have not been characterized as well. Another gene located within the candidate region is LOC_05g02730, which encodes a WUSCHEL-related homeobox 3B. WUSCHEL homeobox genes belong to a family of plant-specific transcription factors that have been shown to carry out specialized functions in key developmental processes in plants, including stem cell fate regulation, embryonic patterning and organ formation (Van der Graaf et al. 2009; Haecker et al. 2004). Expression and functional analysis of WOX3 in Arabidopsis $(P R S)$ and maize (NS1 and NS2) demonstrated that
WOX3 is required in the recruitment of founder cells from the lateral compartment of shoot meristems that will later form the lateral and marginal regions of leaves and leaf orthologues. Null mutations in PRS of Arabidopsis resulted in defects in lateral sepal development and the elimination of the knife-edge cells in the marginal regions of the abaxial and adaxial sepal. Overexpression of the gene, on the other hand, resulted in the formation of multicellular bulges with trichomes on the stem and peduncle of the plant. Such structures were shown to be outgrowths of the epidermal cells (Matsumoto and Okada 2001). In maize, $n s 1$ and $n s 2$ mutations resulted in narrow leaves, with complete deletion of tooth hairs on the margins, as well as stem curvature and shortened internodes (Scanlon et al. 1996).

In our study, complementation tests showed that genomic fragments covering the ORF of dep could restore the formation of bristle type trichomes on the leaf blade and the glumes of glabrous rice, indicating the role of this gene in trichome formation. However, it does not influence the formation of aerial appendages in the leaf margin. Phylogenetic analysis showed that dep groups with the reported WOX3 branch carrying duplications of the PRS/NS branch in the WOX3 lineage. While members of both clades relate to leaf development, PRS/NS orthologues are expressed in the lateral domain whereas those of the WOX3 duplicate clade are expressed in the base of developing phytomers in the shoot meristem (Shimizu et al. 2009; Nardmann et al. 2007; Nardmann and Werr 2006). Such differences in expression domains have been shown to be indicative of subfunctionalization of duplicate genes as a means to escape mutational decay (Nardmann et al. 2007; Lynch and Force 2000). Grouping of dep with the members of the WOX3 duplicate branch suggests that dep may have arisen as a duplicate gene and was preserved by acquiring a sub-function that differs with respect to tissue expression specificity. This may account for the ability of dep to affect trichome formation in the leaf blade and glumes but not in the margins of the leaves. The localization of dep in the nucleus is consistent with the reported features of transcription factors.

Comparative sequence analysis for $\mathrm{dep}$ failed to identify the causal mutation that conditions the glabrous phenotype in rice, suggesting a possible epigenetic regulation of the gene responsible for the trait.

\section{Conclusions}

Despite being a selectively neutral trait, interest in the glabrous phenotype in rice remains due to the practical advantages of cultivating glabrous varieties. In this study, dep encoding a WUSCHEL-related homeobox 3B protein was identified as the gene controlling glabrousness in rice leaves and glumes. The identification and cloning 
of dep will not only provide basis for future research on the elucidation of the molecular mechanisms underlying trichome development (i.e. initiation, distribution and patterning) in rice, but will also facilitate the efficient breeding for glabrous rice varieties using marker-aided selection.

\section{Methods}

\section{Plant materials}

Oryza glaberrima Acc IRGC104038, O. sativa subsp japonica cv. Koshihikari and a chromosome segment substitution line (GLSL15) previously identified as having glabrous leaves and glumes were used in this study (Shim et al. 2010). The plant materials were either grown in the greenhouses of the Laboratory of Plant Molecular Biosystem or under natural conditions in the research field of Nagoya University, Togo, Aichi, Japan. Seedlings of the plant materials were first raised in the greenhouse for 30 days before transplanting them in the field.

\section{Leaf ultrastructure of glabrous rice plants}

The presence or absence of trichomes on the leaves of $1 \frac{1 / 2-}{2}$ or 2-month-old plants was determined by running a forefinger along the length of the leaf blade starting from the tip and going to the base while holding the tip of the leaf. Glabrous leaves offered no resistance to this action due to the absence of bristle type trichomes. To closely examine the leaf ultrastructure of the plant materials, scanning electron microscopy was employed. Leaves of Koshihikari, $O$. glaberrima and GLSL15 were sampled in vials, fixed in FAA (50\% ethanol, formalin and glacial acetic acid at 18:1:1 ratio) solution and dehydrated using a graded series of acetone washes (30, 50, 70, 80, 90, 95, 99 and 100\%). The samples were further dehydrated by critical point drying for $1 \mathrm{~h}$ at $35^{\circ} \mathrm{C}$ and 1200 psi using liquid $\mathrm{CO}_{2}$ as the transitional fluid. Samples were then mounted in a specimen stub, ioncoated and examined under a scanning electron microscope (Hitachi S-2600N). The presence of trichomes on the glumes was determined by manual examination and by checking the hulls under a light microscope.

\section{Linkage analysis and mapping of dep}

To fine-map dep, a total of $600 \mathrm{~F}_{2}$ plants generated by crossing GLSL15 with Koshihikari, as well as $7652 \mathrm{~F}_{3}$ plants generated from $\mathrm{F}_{2}$ lines with informative recombination points, were genotyped using SSRs and newly developed insertion/deletion (indel)-based and derived cleaved amplified polymorphic sequence (dCAPS; Neff et al. 2002) markers targeting the putative location of the gene. Genomic DNA from the mapping population was extracted using the TPS method. SSRs, and indel and dCAPS markers were amplified using standard PCR protocols and run in $3 \%$ agarose gel with ethidium bromide.
Indel and dCAPS markers were designed based on the existing sequence alignment of Nipponbare and 93-11, as well as sequence alignments of the initial $62-\mathrm{kb}$ candidate region for dep in Koshihikari and O. glaberrima. The alignment was generated using the GENETYXMAC/ATSQ (ver. 15.0.5) software.

\section{Complementation tests}

Three sets of complementation tests were carried out parallel to fine mapping to identify the gene controlling glabrousness in rice. Koshihikari BAC (CRC26L16) covering the putative location of the glabrous gene was screened to confirm the presence of markers within the candidate gene region. To develop a sub-library of the clone, the BAC was either completely digested with AscII and BamHI, or partially digested with HindIII to generate differently sized fragments. These fragments were run in 1\% low-melting agarose, eluted using the GELase system (Epicentre Biotechnologies, Madison, WI, USA) and ligated to the binary vector, TAC7. The binary vector was also digested with the same set of enzymes and separately treated with cow intestine alkaline phosphatase (CIAP) to prevent self-ligation. Ligation was performed using the Takara DNA Ligation Kit LONG (Takara, Otsu, Japan), following the manufacturer's specifications. The recombinant plasmid was then transformed to Escherichia coli strain DH10B by electroporation and plated in LB medium with kanamycin. Plasmid DNA extraction followed by digestion using the appropriate enzyme was carried out to verify the presence of an insert. Alongside this, direct plasmid sequencing using the primer pair R3: 5/-AAT TAG GCC CGG GCG GAT-3/ and L3: 5/-GGC CGC GGC CGG CCG TCG -3/ was carried out to estimate the size and determine the map position of the insert. Recombinant plasmids harbouring inserts that cover the candidate gene region were separately introduced to Agrobacterium tumefaciens strain EH105 by electroporation (Hood et al. 1986) and used to transform a glabrous substitution line in cv. T65 background following the methods of Hiei et al. (1994). Transformants were selected in a medium containing $50 \mathrm{mg}$ hygromycin/l. The hygromycin-resistant plants were transplanted in the soil and maintained under greenhouse conditions at an ambient temperature of $30^{\circ} \mathrm{C}$ and $16 \mathrm{~h}$ light: $8 \mathrm{~h}$ dark photoperiod. Sub-clones used for subsequent complementation tests were derived from clones that restored the pubescent phenotype in the glabrous rice donor used for transformation.

\section{Phylogenetic and sequence analysis for dep}

The genomic sequence of dep, along with the 2-kb region upstream of the gene was amplified by PCR from the genomic DNA of O. glaberrima, Koshihikari, glabrous rice mutants in a T65 background and a natural 
population of glabrous and pubescent O. glaberrima lines. The obtained amplicons were purified using the Promega Wizard Genomic DNA Purification Kit, sequenced using the Applied Biosystems 3130xl Genetic Analyzer and analysed using Genetyx-MAC/ATSQ. Orthologues of the translated dep were retrieved by homology searches using Blastx in the National Center for Biotechnology Information and Rice Annotation Project databases. The retrieved protein sequences were aligned by Clustalx ver. 2.0 using the default settings (Larkin et al. 2007). Phylogenetic analysis based on the Neighbor-Joining method was performed using SplitsTree4 ver. 4.11.3 (Huson and Bryant 2006). The accuracy of the inferred phylogenetic relationship was determined by bootstrapping using 1000 replicates.

\section{Subcellular localization}

The coding sequence of dep was ligated into the pA7GFP plasmid vector to construct a dep-GFP fusion protein driven by the cauliflower mosaic virus $35 \mathrm{~S}$ promoter. The constructs were then introduced into onion epidermal cells by particle bombardment. The GFP signal was viewed by confocal laser microscopy.

\section{Promoter-GUS assay}

To determine the spatial expression of dep, the promoter region (including an 80-bp proximal region) of the gene was digested with the appropriate enzymes and fused to the GUS coding sequence via ligation to the pBl101 plasmid vector. The promoter-GUS fusion construct was then digested from the pBl101 plasmid, cloned into Hm12 and transformed to E. coli by electroporation. Recombinant plasmid DNA extracted from $E$. coli was used for transfection in A. tumefaciens that was in turn used for plant transformation. Tissue samples from transgenic plants were stained for $12 \mathrm{~h}$ under dark conditions with X-Gluc solution (5.7 mM 5-bromo-4-chloro-3-indolyl- $\beta$ D-glucuronide, $1.5 \mathrm{mM} \mathrm{K}{ }_{3} \mathrm{Fe}(\mathrm{CN})_{6}, 0.9 \%$ Triton-X 100) before viewing under a stereomicroscope.

\section{Competing interests}

The authors declare that they have no competing interest.

\section{Authors' contributions}

RBAS performed the genetic experiments, transgenic assays, scanning electron microscopy, DNA sequencing and phylogenetic analysis, and wrote the manuscript. KA performed scanning electron microscopy and contributed in the genetic analysis. TT screened, prepared and provided the BAC clones used for plant transformation. JS, TK and MaA contributed in the genetic analysis. MoA conceived and designed the study and critically reviewed the manuscript. All authors read and approved the final manuscript.

\section{Author details}

${ }^{1}$ Bioscience and Biotechnology Center, Nagoya University, Chikusa, Nagoya Aichi 464-8601, Japan. ${ }^{2}$ Honda Research Institute Japan, Kazusa-Kamatari, Kisarazu-shi Chiba 292-0818, Japan. ${ }^{3}$ Present address: Plant Breeding, Genetics and Biotechnology Division, International Rice Research Institute, DAPO Box 7777, Metro Manila, Philippines. ${ }^{4}$ Present address: Upland Farming
Research Division, NARO Hokkaido Agricultural Research Center, 9-4 Shinsei-minami, Memuro, Kasai Hokkaido 082-0081, Japan.

Received: 26 December 2011 Accepted: 27 September 2012 Published: 2 October 2012

\section{References}

Bandyopadhyay T, Gangopadhyay G, Poddar R, Mukherjee KK (2004) Trichomes: their diversity, distribution and density in acclimatization of teak (Tectona grandis L.) plants grown in vitro. Plant Cell Tissue Organ Cult 78:113-121

Barg R, Sobolev I, Eilon T, Gur A, Chmelnitsky I, Shabtai S, Grotewold E, Salts Y (2005) The tomato early fruit specific gene Lefsm1 defines a novel class of plant specific SANT/MYB domain proteins. Planta 221(2):197-211

Foster KW, Rutger JN (1978) Independent segregation of semidwarfing genes and a gene for pubescence in rice. J Hered 69:137-138

Haecker A, Gross-Hardt R, Geiges B, Sarkar A, Breuninger H, Herrmann M, Laux T (2004) Expression dynamics of WOX genes mark cell fate decisions during early embryonic patterning in Arabidopsis thaliana. Development 131(3):657-668

Hauser MT, Harr B, Schlotterer C (2001) Trichome distribution in Arabidopsis thaliana and its close relative Arabidopsis lyrata: molecular analysis of the candidate gene GLABROUS1. Mol Biol Evol 18(9):1754-1763

Hiei Y, Ohta S, Komari T, Kumashiro T (1994) Efficient transformation of rice (Oryza sativa L.) mediated by Agrobacterium and sequence analysis of the boundaries of the T-DNA. Plant J 6:271-282

Hood EE, Helmer GL, Fraley RT, Chilton MD (1986) The hypervirulence of Agrobacterium tumefaciens A281 is encoded in a region of pTiBo542 outside of T-DNA. J Bacteriol 168(3):1291-1301

Hoshikawa Y (1975) Growth of the rice plant. Nosan-Gyoson Bunka Kyokai, Tokyo

Humphries JA, Walker AR, Timmis JN, Orford SJ (2005) Two WD-repeat genes from cotton are functional homologues of the Arabidopsis thaliana TRANSPARENT TESTA GLABRA1 (TTG1) gene. Plant Mol Biol 57(1):67-81

Huson DH, Bryant D (2006) Application of phylogenetic networks in evolutionary studies. Mol Biol Evol 23(2):254-267

Islam MT, Golam Sarwar AKM, Begum HH, Ito T (2009) Epidermal features of rice leaf cv. BRRI Dhan29. Bangl J Plant Taxon 16(2):177-180

Johnson HB (1975) Plant pubescence: an ecological perspective. Bot Rev 41:233-258

Kirik V, Simon M, Huelskamp M, Schiefelbein J (2004) The ENHANCER OF TRY AND CPC1 gene acts redundantly with TRIPTYCHON and CAPRICE in trichome and root hair cell patterning in Arabidopsis. Dev Biol 268(2):506-513

Kobayashi K, Ishikawa R, Senda M, Akada S, Harada T, Niizeki M (1997) Six glabrous phenotypes in rice classified with a scanning electron microscope. Rice Genet Newsl 14:53-55

Larkin MA, Blackshields G, Brown NP, Chenna R, McGettigan PA, McWilliam H, Valentin F, Wallace IM, Wilm A, Lopez R, Thompson JD, Gibson TJ, Higgins DG (2007) Clustal W and Clustal X version 2.0. Bioinformatics 23(21):29472948

Li W, Wu J, Weng S, Zhang D, Zhang Y, Shi C (2010) Characterization and fine mapping of the glabrous leaf and hull mutants (gl1) in rice (Oryza sativa L.) Plant Cell Rep 29(6):617-627

Lynch M, Force A (2000) The probability of duplicate gene preservation by subfunctionalization. Genetics 154(1):459-473

Makenzie KS, Bollich CN, Rutger JN, Kuenzel Moldenhauer KA (1987) Rice. In: Fehr WR, Principles of cultivar development. Crop species, vol 2. McMillan Publishing Company, New York, pp 487-532

Martin C, Glover BJ (2007) Functional aspects of cell patterning in aerial epidermis. Curr Opin Plant Biol 10(1):70-82

Matsumoto N, Okada K (2001) A homeobox gene, PRESSED FLOWER, regulates lateral axis-dependent development of Arabidopsis flowers. Genes Dev 15:3355-3364

Mauricio R, Rausher MD (1997) Experimental manipulation of putative selective agent provides evidence for the role of natural enemies in the evolution of plant defense. Evol 51(5):1435-1444

Moose SP, Lauter N, Carlson SR (2004) The maize macrohairless1 locus specifically promotes leaf blade macrohair initiation and responds to factors regulating leaf identity. Genetics 166(3):1451-1461

Nagao S, Takahashi M (1963) Trial construction of twelve linkage groups in Japanese rice (Genetic studies on rice plant, XXVII). J Fac Agr Hokkaido Univ 53(1):72-130 
Nardmann J, Werr W (2006) The shoot stem cell niche in angiosperms: expression patterns of WUS orthologues in rice and maize imply major modifications in the course of monocot and dicot evolution. Mol Biol Evol 23(12):2492-2504

Nardmann J, Zimmermann R, Durantini D, Kranz E, Werr W (2007) WOX gene phylogeny in Poaceae: a comparative approach to addressing leaf and embryo development. Mol Biol Evol 24(11):2472-2484

Neff MM, Turk E, Kalishman M (2002) Web-based primer design for single nucleotide polymorphism analysis. Trends Genet 18:613-615

Rutger JN, Mackill DJ (2001) Application of Mendelian genetics in rice breeding. In: Khush GV, Brar DS, Hardy B (eds) Rice Genetics IV, Proceedings of the Fourth International Rice Genetics Symposium, Los Baños, Philippines, October 2000. International Rice Research Institute, Philippines, p 29

Sastry MVS, Seetharaman R (1980) Inheritance in rice. Indian J Genet 40(3):573-577

Sato Yl, Sato T, Morishima H (1990) Changing segregation ratios for $\mathrm{gl-1}$ due to unequal chances of fertilization of female gametes. Rice Genet Newsl 7:97

Scanlon MJ, Schneeberger RG, Freeling M (1996) The maize mutant narrow sheath fails to establish leaf margin identity in a meristematic domain. Development 122(6):1683-1691

Serna L, Martin C (2006) Trichomes: different regulatory networks lead to convergent structures. Trends Plant Sci 11(6):274-280

Shimizu M, Al Mamun A, Naito K (1976) A study on the rice leaf trichome with special reference to varietal differences. Crop Sci Soc Jpn 76:29-32

Shimizu R, Ji J, Kelsey E, Ohtsu K, Schnable PS, Scanlon MJ (2009) Tissue specificity and evolution of meristematic WOX3 function. Plant Physiol 149(2):841-850

Shim RA, Angeles ER, Ashikari M, Takashi T (2010) Development and evaluation of Oryza glaberrima Steud. chromosome segment substitution lines (CSSLS) in the background of O. sativa L. cv. Koshihikari. Breed Sci 60:613-619

Suo J, Liang X, Pu L, Zhang Y, Xue Y (2003) Identification of GhMYB109 encoding an R2R3 MYB transcription factor that expressed specifically in fiber initials and elongating fibers of cotton (Gossypium hirsutum L.). Biochim Biophys Acta 1630(1):25-34

Szymanski DB, Lloyd AM, Marks MD (2000) Progress in the molecular genetic analysis of trichome initiation and morphogenesis in Arabidopsis. Trends Plant Sci 5(5):214-219

Tominaga R, Iwata M, Sano R, Inoue K, Okada K, Wada T (2008) Arabidopsis CAPRICE-LIKE MYB 3 (CPL3) controls endoreduplication and flowering development in addition to trichome and root air formation. Development 135(7):1335-1345

Van der Graaf E, Laux T, Rensing SA (2009) The WUS homeobox-containing (WOX) protein family. Genome Biol 10(12):248

Varnamkhasti MG, Mobli H, Jafari A, Keyhani AR, Soltanabadi MH, Rafiee S, Kheiralipour K (2008) Some physical properties of rough rice (Oryza sativa L.) grain. J Cereal Sci 47(3):496-501

Wada T, Tachibana T, Shimura Y, Okada K (1997) Epidermal cell differentiation in Arabidopsis determined by a Myb homolog, CPC. Science 277:1113-1116

Wang S, Hubbard L, Chang Y, Guo J, Schiefelbein J, Chen JG (2008) Comprehensive analysis of single repeat R3 MYB proteins in epidermal cell patterning and their transcriptional regulation in Arabidopsis. BMC Plant Biol 8:81

Wang S, Wang JW, Yu N, Li CH, Luo B, Guo JY, Wang L, Chen XY (2004) Control of plant trichome development by a cotton fiber MYB gene. Plant Cell 16(9):2323-2334

Werker E (2000) Trichome diversity and development. Adv Bot Res 31:1-35 Yamamoto T, Nishikawa A, Nakajima Y, Oeda K, Hirohara H (1997) Genetic and morphological studies on glabrousness of a somaclonal variant induced by anther culture in rice (Oryza sativa L.). Breed Sci 47:1-6

Yanhui C, Xiaoyuan Y, Kun H, Meihua L, Jigang L, Zhaofeng G, Zhiqiang K, Yunfei Z, Xiaoxiao W, Xiaoming Q, Yunping S, Li Z, Xiaohui D, Jingchu L, Xing-Wang D, Zhangliang C, Hongya G, Li-Jia Q (2006) The MYB transcription factor superfamily of Arabidopsis: expression analysis and phylogenetic comparison with the rice MYB family. Plant Mol Biol 60(1):107-124

Yu ZH, Kinoshita T, Sato S, Tanksley SD (1992) Morphological and RFLP markers are associated in rice. Rice Genet Newsl 9:116-118

Yu ZH, McCouch SR, Kinoshita T, Sato S, Tanksley SD (1995) Association of morphological and RFLP markers in rice (Oryza sativa L.). Genome 38(3):566-574
Zhao M, Morohashi K, Hatlestad G, Grotewold E, Lloyd A (2008) The TTG1-bHLHMYB complex controls trichome cell fate and patterning through direct targeting of regulatory loci. Development 135(11):1991-1999

\section{doi:10.1186/1939-8433-5-28}

Cite this article as: Angeles-Shim et al:: A WUSCHEL-related homeobox 3B gene, depilous (dep), confers glabrousness of rice leaves and glumes. Rice 2012 5:28.

\section{Submit your manuscript to a SpringerOpen ${ }^{\odot}$ journal and benefit from:}

- Convenient online submission

- Rigorous peer review

- Immediate publication on acceptance

- Open access: articles freely available online

- High visibility within the field

- Retaining the copyright to your article

Submit your next manuscript at $\gg$ springeropen.com 